\title{
Nonintestinal-Type Sinonasal Adenocarcinoma
}

Malignant glandular neoplasms of the sinonasal tract originate either from the respiratory epithelium or the underlying mucoserous glands. They present with a confusing array of morphologic features and this is reflected in the nomenclature of these tumors. These tumors are grouped into three main types: salivary gland-type, intestinaltype and non-intestinal type adenocarcinomas.' Salivary gland-type adenocarcinomas of the nasal cavity histologically resemble their analogous lesions in the major and minor salivary glands. Adenoid cystic carcinoma is the most common although almost any of those described in the salivary glands can occur in the nasal cavity as well. Intestinaltype adenocarcinomas resemble glandular neoplasms that occur in the small and large intestines. The more well-differentiated ones resemble colonic tubular and villous adenomas while those at the other end of the spectrum resemble moderately to poorlydifferentiated colonic adenocarcinomas. Others may be composed of goblet cells or resemble colonic mucinous carcinomas. Nonintestinal-type adenocarcinomas are the most diverse of the lot and are composed of adenocarcinomas whose morphologies do not easily fit in into the previous two categories. For purposes of prognostication, they are divided into low-grade and high-grade categories based on architecture, nuclear features and mitotic activity. Low-grade tumors have uniform cells arranged in compact acini, back to back, confluent glands, cystic spaces and papillae. They maintain tall columnar to cuboidal arrangements without much stratification. Cytoplasm is often abundant but variable in appearance - basophilic, granular, mucinous, eosinophilic and also oncocytic. Nuclear atypia is mild to moderate with few mitoses. High-grade tumors are mostly solid, show prominent nuclear pleomorphism, nucleoli and mitotic activitiy. Signet-ring cells may be seen. Necrosis may often be present. ${ }^{2,3,4}$

We present the case of a 73-year-old female with a destructive left nasal cavity mass. Biopsy shows an infiltrative epithelial tumor with a papillary configuration composed of tumor cells draped around vascular cores (Figures 1 and 2). High-power view shows cuboidal to polygonal cells that have large, angular and hyperchromatic nuclei without distinct nucleoli. Mitoses are difficult to come by. Cytoplasm is moderate to abundant and has a dense eosinophilic, somewhat oncocytic quality (Figures 3 and 4). Mucinsecreting or other intestinal-type cells are not seen. The case was signed out as a lowgrade, papillary, nonintestinal-type adenocarcinoma. Unfortunately, the patient was subsequently lost to follow-up.

Philipp J Otolaryngol Head Neck Surg 2009; 24 (2): 41-42 


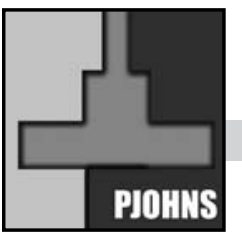

\section{UNDER THE MICROSCOPE}

Philippine Journal Of Otolaryngology-Head And Neck Surgery

Vol. 24 No. 2 July - DeCEMBer 2009

Among patients with intestinal- and nonintestinal-type adenocarcinomas, histologic grade affects outcome. Welldifferentiated tumors with predominantly papillary and tubular configurations do better (80\% 5-year survival) while poorly differentiated ones do poorly (40\% 5-year survival). Recurrences develop in about $50 \%$ and distant metastasis in $15 \%$. Overall survival is about $40 \%$ with death occurring in approximately three years. Treatment is radical surgical resection with postoperative radiotherapy. ${ }^{1}$

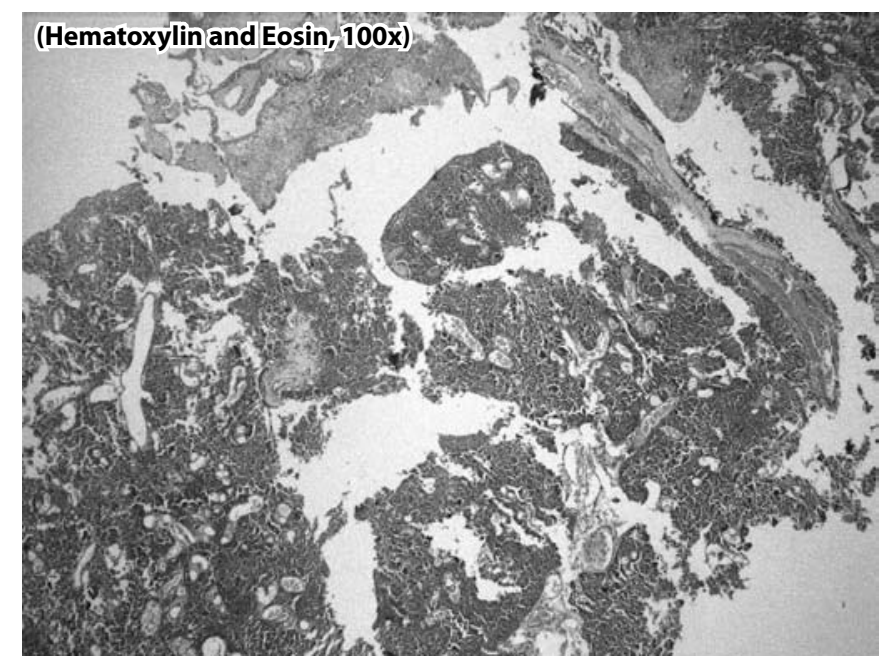

Figure. 1 Infiltrating tumor with a papillary configuration (Hematoxylin and Eosin, 100x).

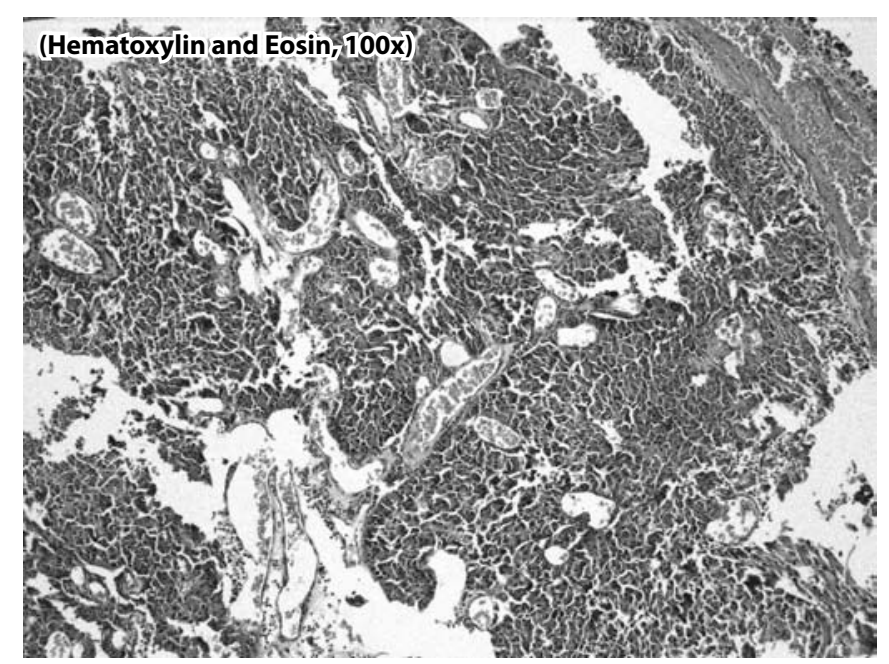

Figure. 2 Infiltrating tumor with a papillary configuration and focally with solid areas. Vascular channels are prominent (Hematoxylin and Eosin, 100x).

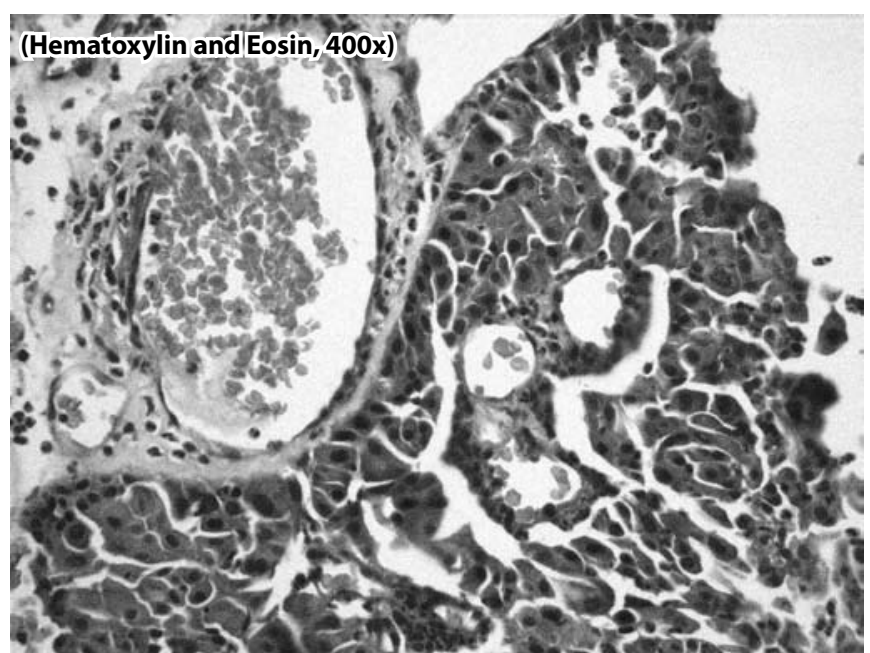

Figure. 3 The tumor cells are cuboidal to polygonal, have large, angular and hyperchromatic nuclei, do not have distinct nucleoli and have rare mitoses (Hematoxylin and Eosin, 400x).

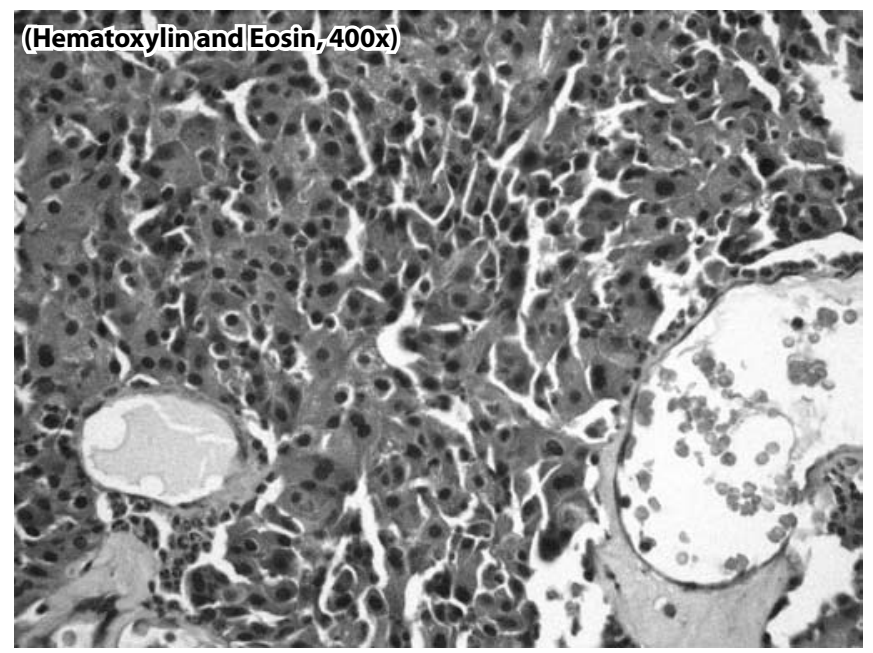

Figure. 4 Cytoplasm is moderate to abundant and has a dense eosinophilic, somewhat oncocytic quality (Hematoxylin and Eosin, 400x). 